\title{
Les marqueurs linguistiques de catégorisation sociale en godié*
}

\author{
Damanan N'dré (Bouaké, Côte d'Ivoire)
}

\begin{abstract}
This article discusses the categorization of people and objects lasses in the Godié society. In Godié, categorization of people and objects is done through classes suffixes. These are the suffixes of nominal classes here called categorization markers. These markers are five. They

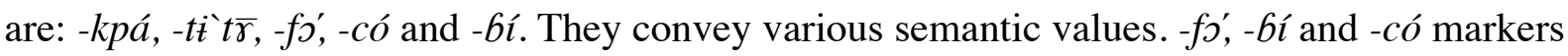
are joined to names of people. They help in categorizing people according to their origin or their provenance in Godié society. $-t \grave{t} t \bar{\gamma}$ and $-k p a ́$, evoke the idea of group. Apart from these markers, the possessives $n^{-}$and $n a$ also have a role to categorize people in the relationship of filiation or alliance.
\end{abstract}

\section{$1 \quad$ Introduction}

Différentes catégories incarnent la société godié. Et il existe des marqueurs linguistiques qui permettent d'établir ces catégories-là. Catégoriser, c'est classer, segmenter et ordonner des éléments en fonction de leur ressemblance, leur similitude, leur identité (Tajfel 1972 ; Tajfel 1974 ; Tajfel/Turner 1986). Sous cet angle, la catégorisation pourrait être définie comme l'action consistant à classer des êtres et/ou des objets selon certaines considérations d'ordre cognitif (Dubois 1991 ; Duboi/Resche-Rigon 1995 ; Dubois/Poitou 2003). La reconnaissance d'un individu, par exemple, à l'appartenance à une catégorie sociale donnée est un acte cognitif majeur. Chaque individu s'identifie à un groupe suivant les affinités qu'il partage avec les autres membres du groupe (Tajfel 1972 ; Tajfel/Turner 1986). Cela résulte d'un attachement profond à des valeurs communes.

Le présent article se propose d'étudier la catégorisation des individus dans la société godié. Dans cette société, le nom est sacré, inaliénable, incorruptible. Son attribution s'accompagne parfois de cérémonies marquant sa sacralité. Chaque famille ou chaque clan a des noms qui lui sont propres et qui ne peuvent être attribués à des tiers sans déviance 'sémantico-cognitive' . Les représentations mentales ayant leur symbolisation dans le langage, il va de soi qu'il ne peut y avoir de dénomination ou de désignation sans la coordination efficiente des entités que sont

\footnotetext{
* Le godié est une langue kru de Côte d'Ivoire (Marchese 1983). Les langues kru sont réparties en deux sousgroupes : ocidental et oriental. Le groupe occidental comprend les langues guéré, grébo, wobé, etc. Le groupe oriental comprend les langues bété, dida, godié, etc. (Marchese 1989). Les langues kru sont rattachées, à l'intérieur du phylum Niger-Congo, au groupe nord Volta-Congo qui comprend aussi les langues gur et les langues Adamawa-Ubanguiennes (Williamson/Blench).
} 
le langage et la cognition (Lakoff/Johnson 1980 ; Lakoff 1986 ; Lakoff 1987 ; Vandeloise 2003).

Les marqueurs dont il est question ici sont en fait des dérivatifs fonctionnant comme des suf-

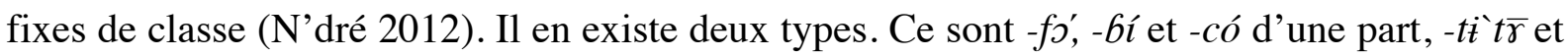
-kpá d'autre part. Les marqueurs -fo', - $6 i$ et -có contribuent à modifier un patronyme pour lui conférer un sens nouveau, passant d'un domaine source à un domaine cible (Lakoff. Ils permettent de catégoriser des individus en fonction de leur origine ou de leur provenance dans la société godié. ${ }^{1}$ Les marqueurs $-t \grave{t} t \bar{\gamma}$ et -kpá ont aussi cette valeur de catégoriser ; mais à la différence des premiers, ils évoquent l'idée de groupe ou d'ensemble. En dehors de ces marqueurs, les déterminants possessifs, dans la langue, ont également pour rôle de catégoriser les individus dans les rapports de filiation ou d'alliance.

Notre démarche se voudrait descriptive, explicative et analytique des faits de langue. Elle permettrait de montrer le fonctionnement morphosyntaxique des différentes constructions obtenues à partir de ces marqueurs. Dans ce qui suit, il s'agira d'examiner d'abord les notions de marqueurs linguistiques et de catégorisation. Il s'agira ensuite d'exposer, de décrire et d'analyser les moyens dont se sert la langue pour catégoriser les individus dans la société. Mais avant, il nous semble important d'exposer, en filigrane, la méthode et la discipline dans laquelle nous entendons situer la présente étude.

\section{Cadre théorique et méthodologique}

\subsection{Cadre théorique}

Nous ne prétendons pas poursuivre un objectif théorique particulier. Nous n'entendons pas non plus tester l'hypothèse d'une école. Nous n'avons pour ambition que de décrire, d'expliquer et d'analyser des faits de langue basés sur la catégorisation des individus à travers certains suffixes de classe. Cet article est donc essentiellement basé sur la présentation de données inédites dont les choix opérés pour définir et caractériser la notion de catégorisation sont soumis à certaines influences théoriques, toutes liées à la cognition humaine, dans ses rapports avec le langage fondamentalement. Ainsi, nous situons la présente étude - en partie et non pas dans son entièreté - dans une perspective générale de la linguistique cognitive. Elle entend montrer, suivant l'objectif commun que s'assignent les différents courants liés à la linguistique cognitive, le fonctionnement et l'appréhension de la signification à travers les liens qui nouent le langage, l'esprit et le cerveau (Lakoff 1980 , 1986 , 1987 ; Langacker 1987 , 1990, 2000 ; Talmy 2000 ; Fuchs 2003 Vandeloise 2003). À cet effet, en dehors des courants cognitivistes d'obédience linguistique, il est fait appel à d'autres disciplines connexes travaillant sur le langage. Il s'agit principalement de la psychologie (Rosch 1973 , 1976 , 1978), de la philosophie (Wittgenstein 1953 ; Austin 1970) et de l'anthropologie (Berlin/Kay 1969). Dans la caractérisation de la notion de catégorisations, toutes ces disciplines aideront à saisir la quintessence de la valeur significative de cette notion.

\footnotetext{
${ }^{1}$ La catégorisation dont il est question ici n'est pas basée fondamentalement sur des considérations discriminatoires au sens où l'entendent les auteurs tels que Tajfel (1974), Taylor (1981) et Billig (1984).
} 


\subsection{Cadre méthodologique}

Les données qui ont servi de base à la présente étude sont des données de première main. Elles ont été recueillies lors d'une enquête lexicale auprès de locuteurs natifs godié. L'enquête a eu lieu dans le département de Fresco, auprès de locuteurs koyo principalement (Kokora 1970). Elle a consisté, suivant l'objectif visé, à répertorier des noms qui ont trait à la classification nominale. D'abord, il était question de situer l'analyse dans un cadre purement morphosyntaxique. Nous nous sommes rendu compte, au fur et mesure que la réflexion prenait forme, que les suffixes de classe dont il est question dans la présente étude introduisaient des considérations d'ordre catégoriel. Ce qui a permis d'orienter l'analyse, en plus du cadre morphosyntaxique générale, dans une perspective de la linguistique cognitive pour une meilleure appréhension de ces suffixes à valeur de catégorisation.

\section{Les notions de marqueur et de catégorisation}

\subsection{Les marqueurs linguistiques}

En linguistique, les marqueurs pourraient être définis, grosso modo, comme des mots grammaticaux ou des expressions liées au discours. Sur cette base, les indices de personnes, dont le rôle est de construire dans le discours, "des identités énonciatives", sont des marqueurs en linguistique. C'est également le cas des indices de temps et de lieu dont le rôle est d'organiser, dans le discours, autour des personnes grammaticales, des relations spatiotemporelles (Benveniste 1966). Ces indices, Benveniste (1966) les définit comme des « marqueurs de subjectivité ». Comme marqueurs en linguistique, nous pouvons citer aussi les déterminants du nom. Leur rôle est d'entretenir avec le nom, dans le discours, des relations d'actualisation. Certaines expressions liées au discours sont appelées « marqueurs discursifs » (Chanet 2003 ; Porhiel 2004 ; Siepmann 2007). Des auteurs, nombreux, considèrent enfin les locutions prépositives telles que "du point de vue, en qualité de, en tant que, dans le cadre de, etc." comme des marqueurs linguistiques dont le rôle est d'introduire dans le discours des catégories syntaxiques ou conceptuelles (Caron 1989 ; Fuchs 1991 ; Chanet 2001 ; Dostie 2004 ; Dostie/Pusch 2007 ; Andersen 2007). Il y a donc autant de définitions qu'il y a d'auteurs s'intéressant à la question de «marqueurs linguistiques ». Pour notre part, les marqueurs dont il est question ici, sont des suffixes de classes qui introduisent dans le nom, l'idée d'une catégorisation des individus dans la société.

\subsection{La notion de catégorisation}

En linguistique cognitive, la catégorisation est dite naturelle (Lakoff 1987). ${ }^{2}$ Elle repose sur des processus perceptuels, expérientiels et conceptuels. Lesquels processus sont capables

\footnotetext{
${ }^{2}$ La notion de catégorisation est une notion transversale. Elle intéresse plusieurs disciplines. Philosophes, anthropologues, psychologues et linguistes se sont depuis toujours intéressés à la question. Aristote, le premier à avoir traité de la question, définit la catégorisation comme le regroupement, à l'intérieur d'une même famille, des individus appartenant à une même classe et ayant le même statut. Cette égalité statutaire des membres d'une même catégorie va être contestée par Wittgenstein (1953) et Austin (1970). Pour eux, tous les membres d'une même catégorie ne peuvent pas avoir le même statut. Chaque membre, sinon certains membres, à l'intérieur de la même catégorie, possèdent des propriétés qui leur sont propres et qui permettent de les distinguer des autres membres.
} 
d'expliquer la complexité du monde physique. Selon Lakoff (1987), à travers les modèles cognitifs idéalisée ou imagés : les ICMs, la connaissance encyclopédique est organisée en termes de structuration abstraite. Elle n'est pas un reflet de la réalité objective tel que nous l'observons directement, mais tel que nous la conceptualisons ou l'appréhendons. Autrement dit, la compréhension de la réalité objective passe nécessairement par un mécanisme cognitif majeur. La théorie de la catégorisation naturelle explique la manière dont la cognition humaine fonctionne en général. Elle explique également la capacité de l'être humain à structurer le monde qui l'entoure à partir des modèles cognitifs idéalisés ou imagés: les ICMs. Cela implique des compréhensions métaphoriques et/ou métonymiques liées à nos croyances et à nos expériences quotidiennes (Lakoff/Johnson 1980 ; Fauconnier 1984 ; Lakoff (1987) et Langacker 1987). Le découpage de la réalité linguistique en plusieurs catégories naturelles est bien un acte cognitif important. En phonologie, en grammaire tout comme en lexicologie et en terminologie, il y a toujours cette répartition catégorielle des faits de langue. Les notions de générique et de spécifique, de défini et d'indéfini (Godé 2008 ; N'dré 2012), d'hypéronymie et d'hyponymie (Kleiber/Tamba 1990), participent de la hiérarchisation des faits langagiers à l'intérieur d'une catégorie linguistique donnée. Il en est de même des notions de massif, de compact, de collectif, de mensuratif (Grinevald 1999), d'animé et d'inanimé, de mâle et de femelle qui sont également un principe de la classification catégorielle des noms d'une langue.

\section{Les moyens linguistiques de catégorisation sociale}

Quand nous parlons de moyens linguistiques de catégorisation sociale, nous faisons allusion à des marqueurs dont le rôle réel s'apparente à celui des suffixes de classes nominales. Ce sont généralement de mots vides de sens en isolation. Ils n'acquièrent de valeur sémantique réelle que suffixés à des mots pleins, dans le sens où l'entend Tesnière (1959). Ils ne concernent qu'un nombre restreint de noms appartenant à des classes nominales différentes. Dans le cadre de la linguistique cognitive et relativement à la notion de catégorisation, ces marqueurs sont définis par rapport aux modèles cognitifs idéalisés ou imagés Lakoff (1986 et 1987). Ils spécifient des propriétés interactionnelles liées à l'expérience et à la culture. Leur emploi dans certaines classes de noms est plus représentatif ou prototypique que dans d'autres.

\section{$4 \quad$ Les marqueurs -tìț̄ et -kpá}

Les marqueurs $-t \grave{t} t \bar{\gamma}$ et -kpá introduisent l'idée de groupe. Mais leur signifié respectif diffère. -kpá évoque l'idée d'un attroupement, d'un regroupement spontané ou non avec absence d'agressivité. Le marqueur -tìt $t \bar{\gamma}$, par contre, évoque l'idée d'un regroupement lent,

Pour les anthropologues tels que Berlin et Kay (1969), la notion de catégorisation trouve son meilleur allié dans l'interaction entre la pensée et le langage. Ils posent en filigrane, à travers leurs études sur les couleurs, la question des universaux linguistiques. Les études menées sur la chromatique, leur ont permis de déduire que chaque peuple organise l'univers à travers ses propres croyances. L'idée qu'il ne saurait y avoir, dans toute catégorie, des membres ayant le même statut est encore défendue par les psychologues. Ainsi, pour Rosch (1973-1976-1978) - dans sa théorie du prototype et de niveau de base - la catégorisation obéit à une hiérarchisation des membres à l'intérieur de la catégorie. Le prototype - noyau central autour duquel s'organise la catégorie - est considéré comme le meilleur exemplaire. 
plus ou moins organisé avec présence d'agressivité ${ }^{3}$. Les deux marqueurs affectent des noms désignant aussi bien des êtres vivants que des entités naturelles. Dès lors, leur statut de marqueurs de catégorisation sociale est mis à mal. Cependant, leurs signifiés respectifs évoquent l'idée de regroupement. Quand par exemple dans le ciel, les nuages s'entassent et deviennent plus dense, il y a un regroupement de nuages qui s'est constitué. Quand des êtres vivants se mettent ensemble spontanément ou non pour une action quelconque commune, il y a également un regroupement qui s'est constitué. - $t \grave{i} t \bar{\gamma}$ et $-k p a ́$ ne sont donc pas à proprement parler des marqueurs de catégorisation. Mais l'idée de catégorisation sociale nous vient à l'esprit dès lors que les termes que ces deux marqueurs affectent ont trait à des êtres vivants et plus particulièrement à des êtres humains. Alors, l'idée d'une catégorisation sociale surgit automatiquement de notre représentation cognitive de la réalité désignée en référence aux modèles cognitifs idéalisés ou imagés de Lakoff (1987); puisque ces deux marqueurs permettent de regrouper des individus dans le but de les discriminer. C'est dans ce sens que nous les considérons, en filigrane, comme des marqueurs de catégorisation sociale. Notons par ailleurs qu'en dehors de -kpá, qui n'a de valeur sémantique réelle que suffixé au terme qu'il détermine, $-\mathrm{t} \grave{i} \mathrm{t} \bar{\gamma}$ peut être employé comme terme de proposition.

\subsubsection{Le marqueur -kpá}

-kpá est un terme ou une expression qui signifie 'ensemble', 'groupe', 'grappe' selon le contexte. Il se suffixe à des noms communs appartenant aussi bien à la classe des humains qu'à la classe des non-humains. Soit en (1), les exemples suivants :

$$
\begin{aligned}
& \text { a. mlā +-kpá } \rightarrow \quad \text { mlā-kpā 'un troupeau' } \\
& \text { 'animaux'+ 'groupe' } \\
& \text { b. nīmlī +-kpá } \rightarrow \text { nīmlì-kpā 'un essaim d'oiseaux' } \\
& \text { 'oiseaux'+'groupe' } \\
& \text { c. jîkpā +-kpá } \rightarrow \text { jīkpà-kpā 'un attroupement' } \\
& \text { 'hommes'+ 'groupe' }
\end{aligned}
$$

En (1a) comme en (1b) ou en (1c), -kpá est employé pour désigner des grands ensembles d'individus partageant des traits identitaires communs. Mais -kpá peut être employé dans des cas de noms spécifiques avec toujours l'idée de groupe ou d'ensemble comme l'indiquent en (2) les exemples suivants :
a. $\quad \overline{5}+$-kpá $\rightarrow \quad$ Ђ-kpā $\quad$ 'un troupeau d'éléphants'
'éléphants'+'groupe'
b. GáT + -kpá $\rightarrow$ bálì-kpā 'un troupeau de buffles' 'buffles'+'groupe'
c. màjúŕ + -kpá $\rightarrow$ màjúr̀-kpā 'un essaim de mange-mil' 'mange-mil'+'groupe'
d. jēblíé + -kpá $\rightarrow$ jēblíì-kpá 'un attroupement de jeunes gens' 'jeunes gens'+'groupe'

\footnotetext{
${ }^{3}$ Nous soulignons que le caractère agressif du groupe réside dans sa densité, dans sa compacité, mais également dans le signifié du suffixe lui-même.
} 
Les termes affectés du suffixe - $k p a ́$ sont tous au pluriel. Cela nous permet de dresser, pour plus de clarté dans l'analyse, les correspondances singulier/pluriel comme suit :

(3)

\begin{tabular}{|c|c|c|}
\hline Singulier & Pluriel & sens \\
\hline$m l \bar{\varepsilon}$ & $m l \bar{a}$ & $\operatorname{animal}(\operatorname{aux})$ \\
\hline nìmlè & $n \bar{\imath} m l \bar{\imath}$ & $\operatorname{oiseau}(\mathrm{x})$ \\
\hline$n \bar{\imath} k p \bar{\jmath}$ & $n \bar{\imath} k p \bar{a}$ & homme(s) \\
\hline$l \bar{\varepsilon}$ & $l \bar{s}$ & éléphant(s) \\
\hline bálহ & bálì & bufle(s) \\
\hline màjó & màjúŕ & mange-mil(s) \\
\hline nēblú & nēblié & jeune(s) homme(s) \\
\hline
\end{tabular}

Nous notons que dans la langue, le morphème de pluriel n'a pas une forme régulière et que, par voie de conséquence, aucune logique ne sous-tend le phénomène de la pluralisation dans la langue. Sinon, comment comprendre, entre autres, que certains noms au singulier, ayant la même voyelle en finale de mot, forment différemment leur pluriel ? Comment comprendre également que la même voyelle en finale de mot sert à la fois de morphème au singulier et au pluriel ? Une étude sur le phénomène de la pluralisation dans la langue devrait être menée pour élucider ces interrogations.

Nous soulignons que le signifié de -kpá est quantifiable. La quantification ne concerne pas les individus constituant les ensembles formés à partir de -kpá, mais les ensembles eux-mêmes. En l'espèce, -kpá évoque l'idée d'une pluralisation des ensembles constitués par lui. Les exemples en (1) donnent ainsi au pluriel le résultat suivant :
a. mlā +-kpó $\rightarrow \quad$ mlā-kp $\bar{\mho}^{4} \quad$ 'des troupeaux'
'animaux'+'groupes'
b. nīmlī +-kpú $\rightarrow$ nīmlì-kp̄ 'des essaims d'oiseaux'
'oiseaux'+'groupes'

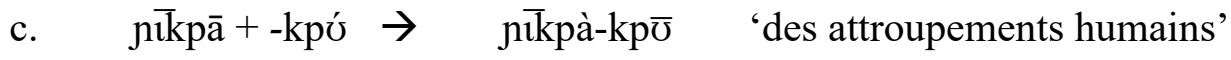
'hommes'+'groupes'

\section{Sens véhiculé par le marqueur -kpá :}

Quand les animaux ou les oiseaux se mettent en groupe, c'est pour traverser des contrées, à la recherche de nourritures. Ils le font de façon spontané et sans agressivité. Un troupeau ou un essaim d'oiseau n'a pour fin que de chercher à se nourrir. Chez les hommes, l'attroupement que suggère le sens de -kpá est au fond le même. En effet, quand des hommes se regroupent ou se mettent ensemble spontanément, c'est pour nourrir leur curiosité. Au cours d'un événement ou d'une situation insolite, par exemple, des individus peuvent se retrouver spontanément pour regarder l'événement ou la situation en question. Curieux de savoir de quoi il est question, chacun y vient pour ne pas se faire conter l'événement. Un attroupement spontané se crée ainsi pour l'occasion. On assiste sans prendre une part active à l'événement ou à la situation du

\footnotetext{
${ }^{4}$ Comme nous venons de le relever avec les exemples en (3), la problématique de la pluralisation dans la langue mérite une étude approfondie. Nous savons, néanmoins, empiriquement, que tout mot ayant en finale de mot la voyelle [a] forme son pluriel à l'aide de la voyelle [u].
} 
moment. C'est généralement le cas, par exemple, des manifestations sportives ou de toute autre activité qui appelle à nourrir la curiosité de chacun. Dans l'un comme dans l'autre cas, le signifié de -kpá suggère l'idée d'un regroupement spontané, non organisé d'individus ayant le même besoin. Dans un tel regroupement, chacun agit sous sa propre responsabilité, n'ayant de compte à rendre à personne. Nous donnons, en guise de résumé, par le graphe ci-dessous, quelques expressions que l'on peut avoir avec le suffixe -kpá :

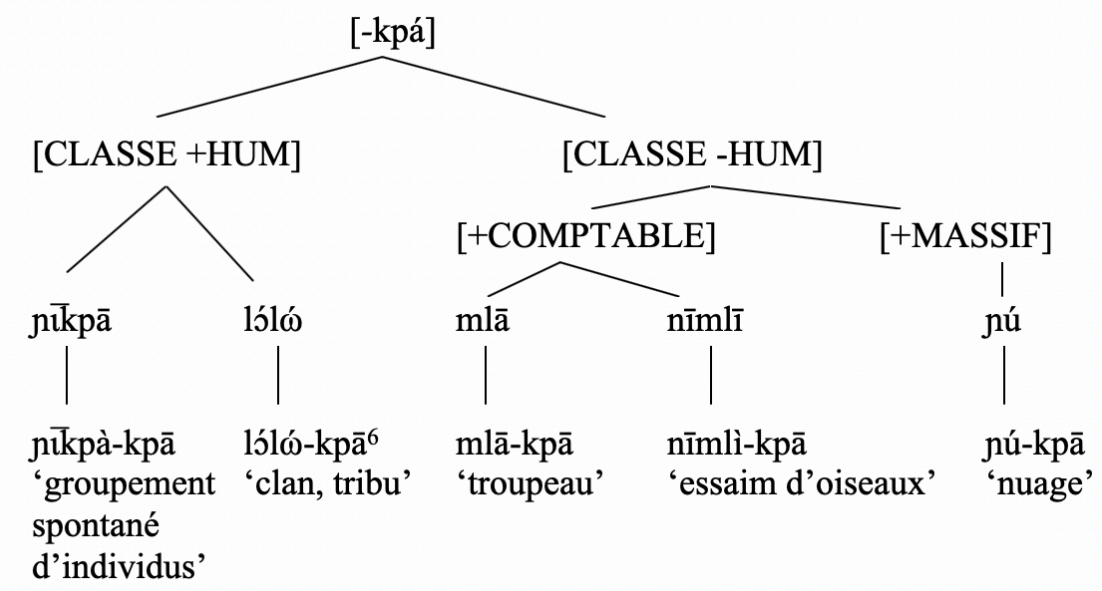

Figure 1: Le suffixe -kpá 5

\section{Commentaire :}

La représentation arborescente ci-dessus a le mérite de donner un aperçu synoptique des différents emplois du marqueur -kpá. Son domaine source semble concerner les animaux et les oiseaux. Ces deux catégories d'êtres sont les plus aptes à un regroupement spontané, guidé par leur instinct de conservation. Le signifié du marque -kpá dans ces deux catégories est plus représentatif ou prototypique. L'homme est moins en crains à ce type de regroupement; et il est complètement inexistant chez les entités et chez les êtres inanimés. Nous disons alors que l'emploi du marqueur -kpá pour caractériser des individus de la classe des humains est d'ordre métaphorique. Il en est surtout des noms de la classe des massifs. Ces deux classes constituent des domaines cibles. Ainsi, la suffixation du marqueur -kpá à des noms de la catégorie des humais ou à des noms de la catégorie des massifs a une valeur hautement métaphorique. Ici, les propriétés de catégorisation des noms, à travers la représentation arborescente, sont d'ordre interactionnelles et sont guidées par l'expérience humaine. La similitude perçue entre ces différentes catégories est donc liée aux modèles cognitifs idéalisés culturellement (Lakoff 1986 et 1987).

\footnotetext{
5 nú-kpā est composé de nú 'eau' et de -kpá 'groupe'. Pour le godié et même généralement, le nuage est constitué par la condensation de vapeurs d'eau. Cognitivement donc, c'est la mise ensemble de différentes vapeurs d'eau qui annonce une éventuelle pluie, dans les limite de l'espace où s'est constituée la condensation. L'appellation $n u ́$ $k p \bar{a}$ pour désigner 'le nuage' trouve tout son sens dans l'interaction entre la langue et la cognition (Vandeloise 2003).
} 


\subsubsection{Le marqueur $-\mathbf{t i t} \bar{\gamma}$}

Le marqueur - tì th̆ à la différence de -kpá, évoque l'idée d'un regroupement, non spontané, lent et plus ou moins organisé avec présence d'agressivité. Le terme est adjoint à des noms d'insectes et de fourmis. C'est généralement le cas avec les termes désignant les fourmis magnans et les abeilles. Les exemples ci-dessous en donnent une illustration :

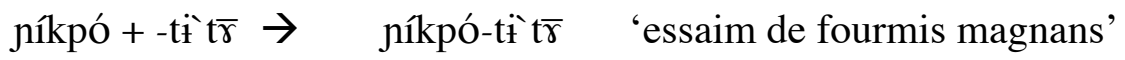

$$
\begin{aligned}
& \text { 'magnans"'+'groupe' }
\end{aligned}
$$

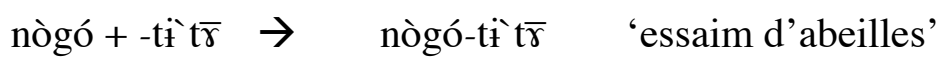

$$
\begin{aligned}
& \text { 'abeilles '+'groupe' }
\end{aligned}
$$

Les essaims d'abeilles et de magnans sont très redoutés. Ces insectes et fourmis se déplacent en colonies. Il s'agit de groupes compacts formant une nappe composée de milliers d'individus très agressifs. Ils imposent le respect et la crainte. Toute espèce animale est tenue en respect lors de leur passage. Si les abeilles sont moins craintes, du fait de leur déplacement dans les airs, les magnans, par contre, sont plus craints, à cause de leur déplacement en rangs serrés sur le sol.

$-t \grave{t} t \bar{\gamma}$ peut également s'adjoindre au terme désignant une pluie torrentielle comme l'indique en (6) l'exemple suivant :

$$
\begin{aligned}
& \text { jú }+ \text { tì } \bar{\gamma} \quad \rightarrow \quad \text { jú-tì } \bar{\gamma}^{6} \quad \text { 'pluie diluvienne/torrentielle' } \\
& \text { 'eau'+'groupe' }
\end{aligned}
$$

Il évoque toujours, dans l'un comme dans l'autre cas, la même idée d'agressivité et de dangerosité. Une pluie diluvienne ou torrentielle est ravageuse, destructrice par l'inondation et l'érosion qu'elle provoque. Le marqueur -tì t⿳亠㐅子 peut enfin s'adjoindre à $n \iota^{-} k p \bar{a}$, pluriel de $n \iota^{-} k p \bar{\partial}$ 'homme' comme l'indique en (8), l'exemple suivant :

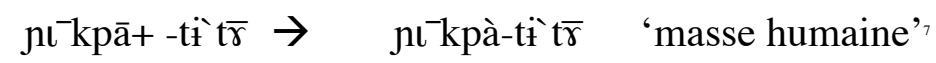

$$
\begin{aligned}
& \text { 'hommes', 'groupe' }
\end{aligned}
$$

Comme en figure (1) nous donnons en figure (2), par le graphe ci-dessous, quelques expressions que l'on peut avoir avec le suffixe $-\mathrm{t} \mathfrak{t} \mathrm{t} \bar{\gamma}$ :

\footnotetext{
${ }^{6}$ Contrairement à nú-kpā qui circonscrit son espace, pour nú-tìţ⿸, l'espace est non circonscrit, plus étendu et la condensation est plus dense et accompagnée de vent violent.

7 Dans cet exemple, c'est un peu redondant de dire jīkpà-tìţ. Le terme tìt $\bar{\gamma}$ suffit à lui seul pour signifier qu'il s'agit d'un groupement humain.
} 


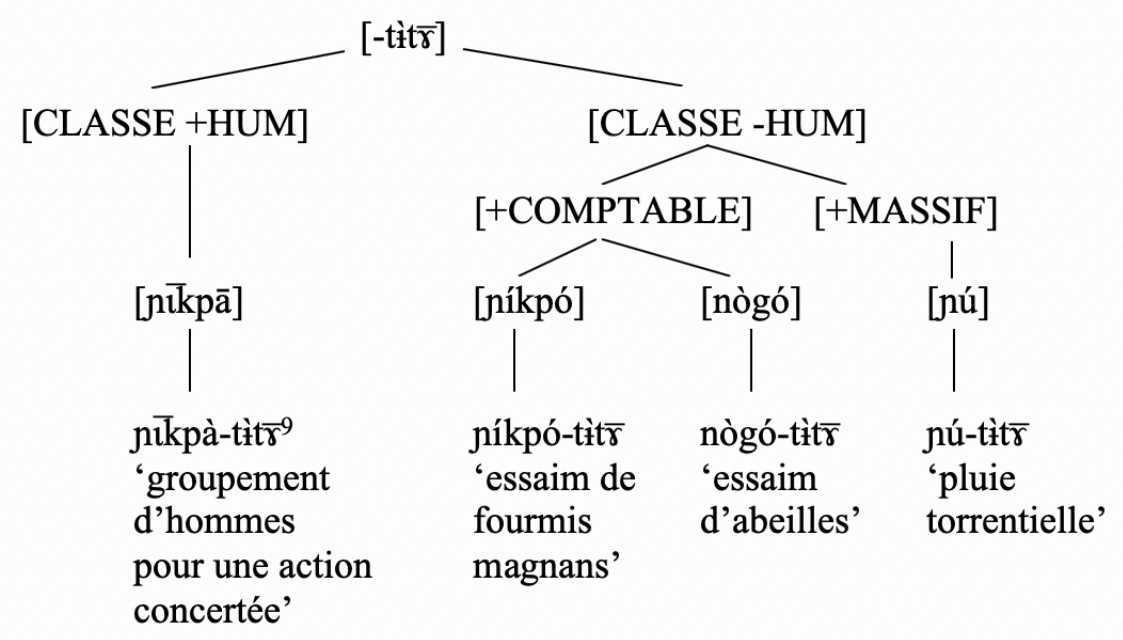

Figure 2: $-t i \grave{t} \bar{\gamma}$

\section{Commentaire :}

Comme en figure (1), la représentation arborescente ci-dessus en figure (2) donne les différents emplois du marqueur $-t \grave{t} t \bar{\gamma}$. Ici, le domaine source semble concerner les magnans et les abeilles. Ces deux catégories d'insectes sont les plus aptes à constituer des masses compactes et denses d'individus redoutables. Nous l'avons dit, le caractère redoutable réside dans la densité du

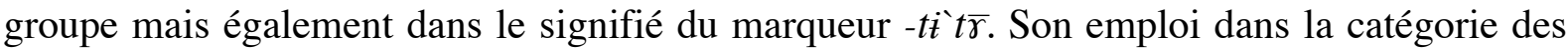
humains comme dans la catégorie des massifs est d'ordre métaphorique et rappelle également les modèles cognitifs idéalisés culturellement (Lakoff 1987). Ces deux catégories constituent des domaines cibles. Comme disent Lakoff et Johnson (1980), la métaphore est une cartographie basée sur l'expérience d'un domaine source vers un domaine cible. Cette cartographie définit une relation entre les modèles cognitifs idéalisés des deux domaines.

\subsection{Les marqueurs -fó, -bí et -có}

Les marqueurs $-f \partial^{\prime}$, $-6 \hat{i}$ et $-c o ́$ sont des dérivatifs. Ce sont des suffixes de classes uniquement réservés à des noms propres de personnes. Considérons en (10) les patronymes suivants :

$$
\begin{aligned}
& \text { wàwàn } \\
& \text { wlígā } \\
& \text { kú̄̄n }
\end{aligned}
$$

A ces patronymes, peuvent être affectés, respectivement, les marqueurs $-f \mathcal{J}^{\prime}$, - $6 \hat{\imath}$ et -có, comme l'attestent en (11) les exemples suivants :

$$
\begin{aligned}
& \text { wàwàn }+-\mathrm{fo}^{\prime} \rightarrow \quad \text { wàwàn-f } \bar{\jmath} \\
& \text { wlígā +-bí } \rightarrow \text { wlígà-6í } \\
& \text { kú̄̄n + -có } \rightarrow \text { kú̄̄n-cō }
\end{aligned}
$$

Ces noms modifiés par la suffixation de ces dérivatifs sont portés par des personnes non autochtones. Les marqueurs $-f f^{\prime}$ et -có se suffixent à des noms portés par des femmes. Le marqueur - $6 i$ se suffixe à des noms portés par des hommes. Chaque suffixe dérivatif véhicule une valeur sémantique précise. 


\subsubsection{Le suffixe $-f{ }^{\prime}$}

Le suffixe $-f \partial^{\prime}$, en isolation, le suffixe porte le ton haut. En construction associative, il est affecté du ton moyen. C'est le cas des noms ci-dessous :

$$
\begin{aligned}
& \text { fàgl̄ }+-f^{\prime} \quad \rightarrow \quad \text { fàglo`-f } \bar{\jmath} \\
& \text { àtēn }+-f^{\prime} \quad \rightarrow \quad \text { àtēn-fō }
\end{aligned}
$$

Ce ton moyen qu'il porte est le résultat d'un processus phonologique. Dans la structure profonde d'une construction associative, il y a un ton bas flottant. Ce ton bas flottant a la capacité d'abaisser d'un cran les tons des termes mis en contribution dans la construction associative, comme c'est le cas de l'exemple suivant :

(11) Jàgl̄ + -fo’ $\rightarrow$ fàgl``-f̄̄

Il s'agit particulièrement du ton de la syllabe finale pour le terme déterminé et le ton de la syllabe initiale pour le terme déterminant (Paradis 1984 ; Somé 2001 ; N'dré 2016). Nous rappelons que dans la langue, la structure de la construction associative est de type déterminé+déterminant.

Toute femme portant un nom affecté du dérivatif $-f f^{\prime}$ est reconnue comme une femme dotée, non ressortissante du village marital. Son intégration passe nécessairement par une cérémonie à laquelle toute la communauté est invitée. C'est au cours de cette cérémonie qu'elle reçoit solennellement le nom qui sera désormais le sien. C'est sous ce nom qu'elle sera désormais désignée. Nul n'a le droit dans le village - sous peine d'amende - de la désigner autrement que par ce nouveau nom. Pour l'homme ou la femme venue d'ailleurs par rapts, aucune cérémonie n'est organisée. Le ravisseur peut donner de son plein gré le nom qu'il veut. Personne ne lui tiendra rigueur dans le village. Cela signifierait-il que le statut accordé à la femme dotée aurait plus de valeur que celui des personnes enlevées par rapt ? Tout porte à le croire. Mais, qu'elles soient enlevées ou dotées, l'intégration de ces personnes, dans la communauté d'accueil, passe nécessairement par un baptême. Le village ayant une valeur virtuelle, c'est par les différents $l \zeta^{\prime} l \omega^{\prime}-k p \sigma^{-}$que se fait concrètement l'intégration. Nous donnons, ci-dessous en (14), quelques noms de femmes dotées, non originaires de l'espace koyo ${ }^{8}$ :

$$
\begin{aligned}
& \text { fàgl̄̄ }+-f^{\prime} \quad \rightarrow \quad \text { fàglo`-fō } \\
& \text { fākpáān }+ \text {-fo' } \rightarrow \quad \text { fākpáān-fō }
\end{aligned}
$$

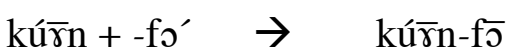

$$
\begin{aligned}
& \text { kpànáān }+- \text { fö }^{\prime} \rightarrow \quad \text { kpànáān-fō } \\
& \text { n } \bar{\gamma} \mathrm{pl} \gamma^{\prime} \bar{\gamma} \mathrm{n}+-\mathrm{fo}^{\prime} \rightarrow \quad \text { j } \bar{\gamma} \mathrm{pl} \gamma^{\prime} \overline{\mathrm{r}} \mathrm{n}-\mathrm{f} \overline{\mathrm{s}} \\
& \text { àtēn }+-f^{\prime} \quad \rightarrow \quad \text { àtēn-fō }
\end{aligned}
$$

\section{Remarque :}

Le suffixe $-f \partial^{\prime}$ ' semble dériver du verbe $f \bar{\jmath}$ qui signifie 'faner'. Les deux termes diffèrent par le ton qu'ils portent respectivement. En isolation, le suffixe porte le ton haut alors que le verbe

\footnotetext{
${ }^{8}$ C'est généralement dans l'espace koyo que les femmes sont baptisées ainsi. Ailleurs, la femme dotée reçoit simplement le nom d'une femme de la famille maritale sans le suffixe en question.
} 
porte le ton moyen. Mais suffixé au nom qu'il détermine, le suffixe est affecté du ton moyen. Le verbe, lui, peut porter le ton bas à l'aspect accompli :

$$
\begin{array}{ll}
\text { mèsī-á fò } & \\
\text { bananes-DEF faner-ACC }
\end{array}
$$

Les bananes ont fané.

ou conserver le même ton moyen avec l'emploi d'un auxiliaire :

$$
\begin{aligned}
& \text { mèsī-á jāa fō } \\
& \text { bananes-DEF AUX faner } \\
& \text { Les bananes ont fané. }
\end{aligned}
$$

NB : Les deux énoncés disent la même chose et signifient littéralement que 'Les bananes ont perdu leur sève'.

S'agit-il de deux termes différents ? Nous pensons que non. Il s'agit du même terme qui prend le sens d'un verbe dans des constructions verbales et le sens d'un dérivatif dans les constructions associatives. L'emploi verbal du terme semble plus représentatif ou prototypique que son emploi de dérivatif nominal. Nous sommes bien dans un contexte de métaphore conceptuelle basée sur l'expérience d'un domaine source vers un domaine cible Lakoff et Johnson (1980). En tant que dérivatif nominal, fo véhicule des valeurs péjoratives. Il attribue au porteur du nom des qualités affectives dévalorisantes. Comme la banane sans sève, il est sans valeur réelle, parce que chosifié. Oui, le verbe puise ses référents dans le domaine des objets.

\subsubsection{Le suffixe $-6 i$}

Le suffixe - $6 i$ semble être un emprunt à la langue gouro. Pris isolement, ce terme n'a aucun signifié dans la langue au contraire du $b l$ ou $b \varepsilon$ du gouro qui signifie 'bras' ou 'branche' (B.T. Irié bi 2016). Du point de vue de la cognition sociale, le gouro considère le fils comme le prolongement du père. Il représente socialement la perpétuation de la lignée. C'est pourquoi, dans la société gouro, le nom de tout enfant de sexe masculin est suivi du morphème [bi]. Par transfert de sens, on pourrait considérer le signifié du suffixe -bí du godié pareillement. Car, généralement, quand le père " adoptif » n'a pas eu d'enfant de sexe masculin, le fils « adopté » devient par voie de conséquence le prolongement de la lignée du père «adoptif». Nous donnons ci-dessous en (17), quelques noms attribués à des hommes arrachés à leurs parents utérins lâchement pour servir de porte-cannes aux ravisseurs :

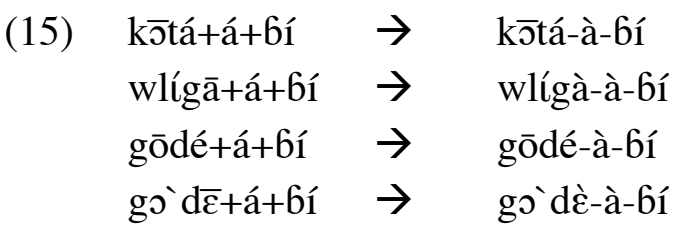

\subsubsection{Le suffixe -có}

Le suffixe -có est un intensificateur de nom. C'est un marqueur qui n'a pas de signifié réel, pris isolement. Il est généralement suffixé au numéral $6 l \bar{o}$ ' 1 ' pour signifier 'seul' : 

(16) àmó blō-có-ò nò lēbè fín moi seul-FOC faire-ACC travail tout C'est moi seul qui ai fait tout le travail.

Suffixé à des noms patronymiques, son signifié semble n'être pas éloigné de sa valeur d'intensificateur. La personne dont le nom est suivi par ce marqueur semble être mis à l'écart. C'est cela que renvoie le signifié de -có cognitivement parlant.

$$
\begin{aligned}
& \text { kú̄̄n+có } \quad \rightarrow \quad \text { kú̄̄n-cō } \\
& \text { dàgo' } \bar{o} n+c o ́ \quad \rightarrow \quad \text { dàgo' } \bar{x} \text {-cōo }
\end{aligned}
$$

Toute femme qui porte un nom affecté du dérivatif -có est reconnue comme une servante, une domestique. Tout homme portant un nom affecté du dérivatif -bí est considéré comme un fils adoptif, un homme de mains. Dans les deux cas, il s'agit de personnes enlevées et ramenées de force hors de leurs habitats. Cela a lieu à l'époque des rapts. Les enfants sont enlevés de force par des ravisseurs pour être leurs hommes de mains. La famille qui les accueille leur attribue des noms pour leur intégration dans la nouvelle société. Ayant été rebaptisées, ces personnes ont plus ou moins les mêmes droits que les personnes originaires du village d'accueil. La citoyenneté de toute personne étrangère dans la société godié est acquise par ce biais. La personne est ainsi intégrée et pourra désormais participer à la vie de la communauté. Tous les droits de la citoyenneté lui sont reconnus et accordés de plein droit. Elle est désormais comptée dans les prises de décisions qui engagent la vie de la communauté.

Pour récapituler, à propos des marqueurs $-f \mathcal{\partial}^{\prime},-6 i$ et -có, nous dressons l'arbre suivant :

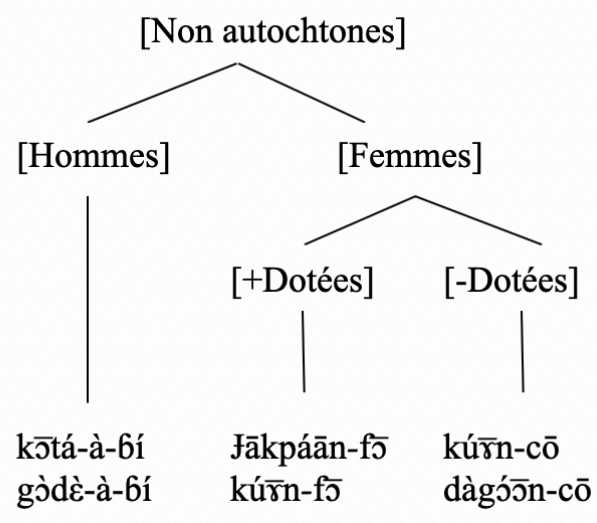

Illustration $3:-f \mathfrak{J}^{\prime},-b i ́$ et $-c o ́$ 


\subsection{Les marqueurs de possession ${ }^{9}$}

Nous appelons ici «marqueurs de possession », les déterminants possessifs. Ils sont de deux ordres. Certains sont qualifiés «d'aliénable» et d'autres «d'inaliénable »10. Dans les liens de filiation ou d'alliance, les possessifs jouent un rôle très important. La forme du possessif varie selon que le lien est considéré comme fort ou lâche. Quand le lien est fort ou étroit, c'est le possessif inaliénable qui est d'usage. Quand il est lâche, c'est le possessif aliénable qui l'est. Soit en (20) les exemples ci-dessous :

$$
\begin{array}{llll}
\text { a. } & \mathrm{n}^{-} & \text {bēlī } & \text { 'mon frère' } \\
\text { b. ná } & \text { bēlī } & \text { 'mon frère' }
\end{array}
$$

Les deux possessifs ont le même sens dénoté. Il s'agit de possessifs de la première personne 'mon/mes'. ${ }^{11}$ Ils déterminent le même nom : 'frère'. Mais ils sont connotés différemment. $n^{-}$ indique que le lien qui unit deux ou plusieurs individus est étroit, inaliénable et incorruptible. Il traduit une proximité, une contiguïté dans les rapports entre les individus. ná, par contre, indique que le lien entre deux ou plusieurs individus est lâche, aliénable et corruptible. Il traduit ainsi une distanciation dans les rapports entre les individus. Ces deux marqueurs possessifs instaurent ainsi dans la société, deux catégories d'individus. Il y a, d'une part, ceux que l'on considère comme proches d'après le lien de consanguinité ou d'alliance et, d'autre part, ceux que l'on considère comme éloignés ; parce que le lien n'est pas consanguin.

\section{Conclusion}

Retenons, en définitive, que la catégorisation sociale, phénomène universel, repose sur des considérations d'ordre cognitif. L'appartenance des individus à telle ou telle communauté participe de ce phénomène. En godié, les moyens linguistiques de catégorisation des individus ou des objets dans la société sont de deux ordres. Il y a ceux qui participent uniquement à la catégorisation des êtres humains ; et ceux qui sont communs aux deux catégories d'êtres ; c'està-dire les êtres humains et les êtres non-humains. Ceux qui participent uniquement à la

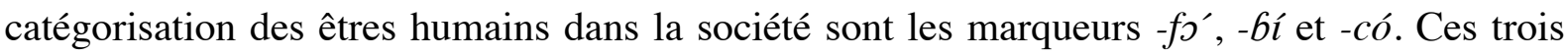
marqueurs, suffixés à des patronymes, indiquent que les individus ainsi nommés ne sont pas originaires de la communauté à laquelle ils appartiennent désormais. Les marques de possession participent également à cette catégorisation. Ils permettent de comprendre, par leur emploi, que tel individu est proche et que tel autre est éloigné. Les moyens linguistiques appliqués à la fois

\footnotetext{
${ }^{9}$ La possession a fait l'objet d'un article qui est en attente de publication. Ainsi, l'objectif visé ici n'est pas de faire un exposé complet du fonctionnement de la possession dans la langue. Nous voudrions simplement montrer, à travers ce chapitre, que dans les relations de filiation ou d'alliance, les marques de possessions établissent des rapports de stratification allant du plus étroit au moins étroit. Du coup, il y a, du point de vue cognitif, l'idée d'une catégorisation des individus par le biais des marques de possession. C'est cela qui a été le motif de ce chapitre.

${ }^{10}$ La possession inaliénable renvoie à un rapport d'inclusion dans lequel les deux composantes de la construction possessive attributive entretiennent une relation de subordination interdépendante, naturelle et permanente (Junker/Martineau 1987 ; Hannon 1988) ; alors que la possession aliénable renvoie à un rapport d'extériorité ou d'appropriation entre les composantes de la construction attributive. Dans celle-ci, la relation qu'entretiennent les composante est accidentelle, non permanente et périssable (Charaudeau 1992 ; Riegel 1984).

${ }^{11}$ Normalement, les possessifs sont invariants du point de vue du nombre. C'est le nom qu'ils déterminent qui oriente le nombre
} 
aux noms appartenant à la classe [+HUMAIN] et aux noms papartenant à la classe [-HUMAIN]

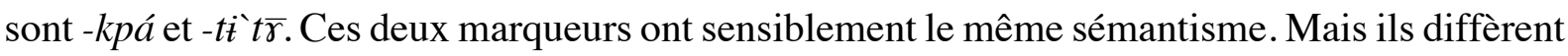
dans la façon dont ils organisent la signification. -kpá indique un regroupement spontané d'individus, non organisé et non agressif. $-t \grave{t} t \bar{\gamma}$ par contre, indique un regroupement d'individus, non spontané, organisé et agressif.

\section{Références bibliographiques}

Andersen, Hanne Leth (2007): « Marqueurs discursifs propositionnels ». Langue française 154: $13-28$.

Austin, John Langshaw (1970) : Quand dire c'est faire. Introduction, traduction et commentaire : Lane, Gilles. Postface : Recanati, François. Paris : Éditions du Seuil.

Benveniste, Emile (1966) : Problème de linguistique générale. Tome 2. Paris : Gallimard.

Berlin, Brent/Kay Paul (1969) : Basic Color Terms: Their Universality and Evolution, Berkeley, University of California Press.

Billig, Maxime (1984) : «Racisme, préjugés et discrimination ». In : Moscovici, Serge (ed.) : Psychologie sociale. Paris, PUF : 449-472.

Caron, Jean (1989) : Précis de psycholinguistique. Paris : PUF.

Chanet, Catherine (2001) : «Connecteurs, particules, et représentations cognitives de la planification discursive ». In: Nemeth, Eniko T. (ed.): Cognition in language use. Selected papers from the 7th International Pragmatics Conference. Vol.1. Antwerp, International Pragmatics Association : 44-55.

Chanet, Catherine (2003) : Fréquence des marqueurs discursifs en français parlé : quelques problèmes de méthodologie. Université de Provence http://www.lpl-aix.fr/ fulltext/1672.pdf [24.07.2016).

Charaudeau, P. (1992) : Grammaire du sens et de l'expression. Paris, Hachette.

Dostie, Gaétane (2004) : Pragmaticalisation et marqueurs discursifs. Analyse sémantique et traitement lexicographique. Bruxelles : De Boeck Duculot.

Dostie, Gaétane/Push, Claus D. (2007) : «Présentation. Les marqueurs discursifs. Sens et variation ». Langue française $154: 3-12$.

Dubois, Danièle (1991) : Sémantique et cognition - catégories, prototypes, typicalité. Paris : CNRS.

Dubois, Danièle/Poitou, Jaques (2003) : « Des «normes catégorielles » : structuration cognitive et/ou linguistique des catégories sémantiques ». Intellectica $35: 217-249$.

Dubois, Danièle /Resche-Rigon, Philippe (1995) : «De la «naturalité» des catégories sémantiques : des catégories «d'objets naturels» aux catégories lexicales ». Intellectica $20: 217$ 245.

Fauconnier, Gilles (1984) : Les espaces mentaux. Paris : Editions du Seuil.

Lakoff, George/Johnson, Mark (1980) : Metaphors We Live By, trad. Les métaphores dans la vie quotidienne. Paris : Les Editions de Minuit.

Fuchs, Catherine (1991) : «Polysémie, interprétation et typicalité : l'exemple de pouvoir ». In Dubois, Danièle (ed.) : Sémantique et cognition - catégories, prototypes, typicalité. Paris. CNRS : 161-170.

Godé, Victor (2008) : Le dadjriwalé, langue kru de la Côte d'Ivoire. Paris: Harmattan. 
Grinevald, Colette (1999) : « Typologie des systèmes de classification nominale ». Faits de Langues 14 : 101-123. DOI : 10.3406/flang.1999.1271.

Hanon, Suzanne (1988) : «Qui à quoi ? Reflexion sur la possession inaliénable et le verbe avoir en français ». Revue Romane 23/2 : 161-177.

Irié Bi Tié, Benjamin (2016) : «Le Système des Anthroponymes Gouro, Langue Mandé-Sud de Côte d'Ivoire : de L'Expression des Valeurs Culturelles Intrinsèques à l'Intrusion de la Diversité Linguistique » Littérature, langues et linguistique. Revue du CAMES 4 : 137-150.

Junker, Marie-Odile/Martineau, France (1987) : « Les possessions inaliénables dans les constructions objet ». Revue Romane 22/2 : 194-209.

Kokora, Dago Pascale (1970) : «Aperçu grammatical du koyo ». Annales de l'université d'Abidjan : 97-113.

Lakoff, George. (1986) : « Classifiers as a Reflection of Mind». In: Craig, Colette (ed.): Noun Classes and Categorization. Amsterdam/Philadelphia, Benjamins: 13-51. (= Typological Studies in Language 7).

Lakoff, George (1987) : Women, Fire, and Dangerous Things: What Categories Reveal about theMind. Chicago/ London : University of Chicago Press.

Langacker, Ronald W. (1986) : «An Introduction to Cognitive grammar ». Cognitive Science 10: $1-40$.

Langacker, Ronald W. (1987) : Foundations of Cognitive Grammar. Vol 1: Theoritical Perequisites. Stanford: Stanford University Press.

Langacker, Ronald W. (1991) : Foundations of Cognitive Grammar. Vol 2 : Descriptive Application. Stanford: Stanford University Press.

Langacker, Ronald W. (2000) : Grammar and Conceptualization. Berlin : de Gruyter.

Marchese, Lynell (1983): Atlas linguistique kru. Abidjan: Institut de Linguistique Appliquée (ILA).

Marchese, Lynell (1989): "kru". In: Bendor-Samuel, John (éd.) (1989): The Niger-Congo languages. New York, Lanham/London, University Press of America: 119-139.

N'dré, Damanan J. (2012) : « Classes nominales et deixisะ : cas des suffixes de pluriel -sı et -nyi du dadjriwale ». Linguistik online 56, 6/12:65-80.

N'dré, Damanan J. (2016) : «Rapport ton/consonne et ton bas flottant dans les constructions associatives du dadjriwalé », Langues et langages en contextes africains: Revue algérienne des sciences du langage $3: 87-111$.

Paradis, Carole (1984) : «Le comportement tonal des constructions associatives en wobe ». Journal of African Languages and Linguistics 6:147-171.

Porhiel Sylvie (2004) : «Les introducteurs thématiques », Cahiers de Lexicologie 85/2 : 9-45.

Riegel, Martin (1984) : «Pour une redéfinition linguistique des relations dites de «possession » et « d'appartenance» ». L'information grammaticale $23: 3-7$.

Rosch, Eleanor (1973) : « Natural Categories », Cognitive Psychology 4/3 : 328-350.

Rosch, Eleanor (1976a) : «Classifications d'objets du monde réel : origines et représentations dans la cognition » Bulletin de psychologie 307-313 : 242-250.

Rosch, Eleanor et al. (1976b) : «Basic objects in natural categories ». Cognitive psychology 8: 382-439.

Rosch, Eleanor (1978a) : «Principles of categorization ». In: Rosch, Eleanor/Lloyd, Barnbara (eds.): Cognition and categorization. Hillsdale, Erlbaum: 27-48. 
Rosch, Eleanor (1978b) : «Human categorization ». In: Warren, Neil (ed.): Advances in cross culture Psychology. London, Academic-press: 4-49.

Siepmann, Dirk (2007) : «Les marqueurs de discours polylexicaux en français scientifique », Revue française de linguistique appliquée 2/2007 (Vol. XII) : 123-136.

Somé, Penou-Achille (2001) : «Tout ton flottant bas autorise-t-il la propagation du ton haut précédent ? » Studies in African Linguistics 30/2 : 195-236.

Tajfel, Henri (1972) : «La catégorisation sociale ». In : Moscovici, Serge (éd.) : Introduction à la psychologie sociale. Paris, Larousse : 272-302.

Tajfel, Henri (1974) : « Social identity and intergroup behavior». Soc. Sci. Inf. 13: 65-93.

Tajfel, Henri/Turner, John C. (1986) : " The social identity theory of intergroup behavior ». In: Worchel, Stephen/Austin, William G. (éds): Psychology of intergroup relations. Chicago, Nelson-Hall: 7-24.

Talmy, Leonard (2000) : Towards a Cognitive Semantics. 2 vol. Cambridge : M.I.T. Press.

Taylor, Shelley E. (1981) : «A categorization approach to stereotyping ». In: Hamilton, David L. (éd.): Cognitive Processes in Stereotyping and Intergroup Behavior. Hillsdale/NJ, Erlbaum: 83-114.

Vandeloise, Claude (2003) : Langues et cognition. Paris : Hermès.

Williamson, Kay/Blench, Roger (2000) : «The Niger-Congo languages: A classification and description of Africa's largest language ». In: Heine, Bernd/Nurse, Derek (éds.) : African Languages - An Introduction. Cambridge, Cambridge University Press: 11-42.

Wittgenstein, Ludwig (1953) : Philosophical investigations. (Oxford: Basil Blackwell. 